\section{Commentary: Winning the match: Hitting the perfect wedge}

\author{
Scott J. Swanson, MD
}

The article by Kamel and colleagues ${ }^{1}$ examining the results of resection for screening-detected lung cancer identified in the National Lung Cancer Screening Trial is another piece in the puzzle of what the role of sublobar resection is in treating lung cancer. The authors make the case that because the biology of lung cancer found through a detection program may be less aggressive, that it is the perfect tumor to be treated using a lesser resection. Their analysis of the data bears this out. They find no statistically significant difference in survival between sublobar resection (mostly wedge resection) and lobectomy for stage I lung cancer. At the same time, morbidity was significantly less in the sublobar group. As with any other large multi-institution database, many important details are missing. We do not have data about number of nodes removed or what stations were accessed. There is no information about how the wedge resection or segmentectomy was performed. We do not know about the length of the margins, the presence of spread-through-air-spaces, or subtype of the non-small cell lung cancer. Thus, we again are faced with the Achilles' heel of the lobectomy versus sublobar resection debate. The 2 crucial issues are: What are the appropriate size, location, and biology of the tumor suitable for sublobar resection? and, How should the operation be performed to obtain the intended results? The results of this article suggest that despite the lack of data mentioned we still find comparable outcomes. However, for wedge resection to be acceptable, in general, for the treatment of small lung cancers $(2.0 \mathrm{~cm}$ or smaller), these details will be necessary to assure our

\footnotetext{
From the Division of Thoracic Surgery, Department of Surgery, Brigham and Women's Hospital, Harvard Medical School, Boston, Mass.

Disclosures: The author reported no conflicts of interest.

The Journal policy requires editors and reviewers to disclose conflicts of interest and to decline handling or reviewing manuscripts for which they may have a conflict of interest. The editors and reviewers of this article have no conflicts of interest.

Received for publication Aug 4, 2021; revisions received Aug 4, 2021; accepted for publication Aug 9, 2021; available ahead of print Aug 12, 2021.

Address for reprints: Scott J. Swanson, MD, Division of Thoracic Surgery, Brigham and Women's Hospital, 75 Francis St, Boston, MA 02115 (E-mail: sjswanson@ bwh.harvard.edu).

J Thorac Cardiovasc Surg 2022;163:1916

0022-5223/\$36.00

Copyright $(2021$ Published by Elsevier Inc. on behalf of The American Association

for Thoracic Surgery

https://doi.org/10.1016/j.jtcvs.2021.08.017
}

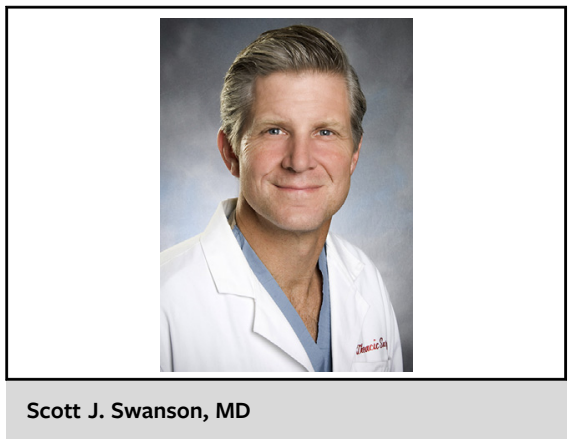

CENTRAL MESSAGE

Sublobar resection when done properly is a good lung cancer operation for small-size lung cancer tumors.

patients that they are receiving the best procedure. The randomized study from Japan only evaluates segmentectomy and the US trial has both segmentectomy and wedge resection. However the US trial does not provide some of the details mentioned above. Going forward, it is reassuring to know that for certain small-size lung cancer tumors, in this case a screening-detected, sublobar resection-particularly wedge resection-is a good, safer option for patients. As we continue to identify these tumors at a smaller size, study their biology, and emphasize the importance of a comprehensive nodal dissection of the draining and mediastinal nodes and achieving a wide margin $(1.0-2.0 \mathrm{~cm})$ as $\mathrm{we}^{2,3}$ and others have published, wedgectomy-the operation that has always had a bad reputation-will assume an important place in our surgical armamentarium.

\section{References}

1. Kamel MK, Lee B, Harrison SW, Port JL, Altorki NK, Stiles BM. Sublobar resection is comparable to lobectomy for screen-detected lung cancer. $J$ Thorac Cardiovasc Surg. 2022;163:1907-15.

2. Mohiuddin K, Haneuse S, Sofer T, Mentzer SJ, Sugarbaker DJ, Swanson SJ. Relationship between margin distance and local recurrence among patients undergoing wedge resection for small $(\leq 2 \mathrm{~cm})$ non-small cell lung cancer. J Thorac Cardiovasc Surg. 2014;147:1169-75.

3. Wolf AS, Richards WG, Jaklitsch MT, Bueno R, Sugarbaker DJ, Swanson SJ. Lobectomy versus sublobar resection for small ( $2 \mathrm{~cm}$ or less) non-small cell lung cancers. Ann Thorac Surg. 2011;92:1819-23. 